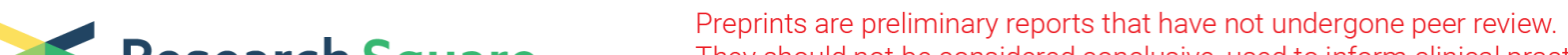 Research Square
or referenced by the media as validated information.
}

\section{Overexpression of a type 2 diacylglycerol acyltransferase in the marine diatom Phaeodactylum tricornutum enhances lipid production and omega-3 Long Chain Polyunsaturated Fatty Acid content}

Richard P. Haslam

Rothamsted Research

Mary L. Hamilton

Rothamsted Research

Chloe K. Economou

Queen Mary University of London

Richard Smith

Rothamsted Research

Kirsty L. Hassall

Rothamsted Research

Johnathan A. Napier

Rothamsted Research

Olga Sayanova ( $\sim$ olga.sayanova@rothamsted.ac.uk)

Rothamsted Research https://orcid.org/0000-0002-8484-2322

\section{Research}

Keywords: Acyl-CoA:diacylglycerol acyltransferase (DGAT), eicosapentaenoic acid, docosahexaenoic acid, triacylgycerol, omega-3 fatty acids, Phaeodactylum tricornutum

Posted Date: January 22nd, 2020

DOI: https://doi.org/10.21203/rs.2.21513/v1

License: (1) This work is licensed under a Creative Commons Attribution 4.0 International License. Read Full License

Version of Record: A version of this preprint was published at Biotechnology for Biofuels on May 14th, 2020. See the published version at https://doi.org/10.1186/s13068-020-01726-8. 


\section{Abstract}

Background: Oleaginous microalgae represent a valuable resource for the production of high value molecules. Considering the importance of omega-3 long chain polyunsaturated fatty acids (LC-PUFAs) for human health and nutrition the yields of high value eicosapentaenoic acid (EPA) and docosahexaenoic acid (DHA) require significant improvement to meet demand, however, the current cost of production remains high. A promising approach is to metabolically engineer strains with enhanced levels of triacylglycerols (TAGs) enriched in EPA and DHA.

Results: Recently we have engineered the marine diatom Phaeodactylum tricornutum to accumulate enhanced levels of DHA in TAG. To further improve the incorporation of omega-3 LC-PUFAs in TAG we focused our effort on the identification of a type 2 acyl-CoA:diacylglycerol acyltransferase (DGAT) capable of improving lipid production and the incorporation of DHA in TAG. DGAT is a key enzyme in lipid synthesis. Following a diatom based in vivo screen of candidate DGATs, a native $P$. tricornutum DGAT2B was taken forward for detailed characterisation. Expression of DGAT2B in P. tricornutum was confirmed by qRT-PCR and the transgenic strain grew successfully in comparison to wildtype. PtDGAT2B has broad substrate specificity with preferences for $\mathrm{C} 16$ and LC-PUFAs acyl groups. Moreover, the expression of DGAT2B resulted in higher lipid yields and enhanced levels of DHA in TAG. Furthermore, co-expression with a $\Delta 5$-elongase showed how iterative metabolic engineering can be used to increase DHA and TAG content, irrespective of nitrogen treatment.

Conclusion: This study provides further insight into lipid metabolism in P. tricornutum and suggests a metabolic engineering approach for the efficient production of EPA and DHA in microalgae.

\section{Background}

Microalgal strains with a high lipid content, stored as triacyglycerol (TAG), are considered a valuable food source and a promising feedstock to produce high value molecules [1]. As a result, research into the pathways that regulate TAG composition and accumulation have received much attention $([2,3]$ and references therein). Often microalgae accumulate high quantities of TAGs in response to abiotic stress e.g. nutrient starvation, high temperature, salinity, $\mathrm{pH}$ or light intensity [4]. The application of such environmental stresses can be an effective strategy for increasing lipid production in microalgae, however their negative effect on cell growth is a major bottleneck for reducing production costs. Therefore, new efficient strategies are needed to increase economic viability of production [5]. A promising approach is the use of genetic engineering for generating improved strains with desirable characteristics such as fatty acid (FA) composition, enhanced lipid biosynthesis and high growth rates.

Within the complex network of activities associated with TAG assembly, acyl-CoA: diacylglycerol acyltransferases (DGATs) and phospholipid:diacylglcerol acyltransferases (PDATs) have been shown to play an important role in TAG accumulation (for reviews see [1] and [6]). DGAT (EC 2.3.1.20) catalyses the final committed reaction in the acyl-CoA dependent Kennedy TAG biosynthetic pathway by the 
esterification of acyl-CoA to a diacylglycerol (DAG) moiety [7, 1]. At least three classes of DGATs have been identified in eukaryotes. Of these, two types, designated DGAT1 and DGAT2, are membrane-bound acyltransferases and play a major role in TAG biosynthesis. Whereas DGAT3 is a soluble isoform characterised in peanut (Arachis hypogaea) and Arabidopsis thaliana, where it is thought to regulate the flux between the cytosolic acyl-CoA pool and TAG $[1,8,9]$. The pivotal role of DGAT in TAG synthesis has been demonstrated by the increase in seed oil content and altered FA composition resulting from the overexpression of plant DGAT1 and DGAT2s (for Refs see $[1,10,11]$ ). Therefore, one beneficial route for the metabolic engineering of microalgae for increased oil content and tailored FA composition may be the manipulation of microalgal DGAT activities.

Most eukaryotic organisms contain at least one copy from the DGAT family of three classes [1]. To date, genome sequencing of photosynthetic microalgae has revealed the presence of a single gene copy encoding the type 1 DGAT, apart from Nannochloropsis oceanica IMET1 where two isoforms were identified [6]. By contrast, multiple copies of DGAT2 genes have been annotated in microalgal genomes, for example five DGAT2s have been found in Phaeodactylum tricornutum and 12 copies in N. oceanica $[12,13]$. Despite this diversity, currently only a few microalgal DGATs have been functionally characterized. In most instances attempts to functionally characterise DGAT isoforms have used a Saccharomyces cerevisiae mutant strain (H1246) lacking endogenous DGAT and PDAT genes. Using this approach DGAT1 isoform from $P$. tricornutum was successfully characterised, demonstrating a preference to produce TAGs with a high level of saturated fatty acids (16:0 and 18:0) [12]. Numerous DGAT2 genes have been putatively identified in microalgae e.g. N. oceanica, Chlamydomonas reinhardtii, Ostreococcus tauri, Thalassiosira pseudonana and P. tricornutum, for subsequent characterisation in TAG deficient $S$. cerversiae $[14,15,16,17,18,19]$. Not all the genes tested restored the TAG phenotype, but of those that did, many showed differing substrate specificity for mono- and polyunsaturated acyl-CoA substrates, e.g. O. tauri DGAT2B showed broad substrate specificity, accepting saturated as well as monoand polyunsaturated acyl-CoAs as substrates [18], while DGAT3 isoform from P. tricornutum, (PtDGAT3) displayed a preference for the incorporation of unsaturated C18-fatty acids [20].

In P. tricornutum four putative type 2 DGATs, annotated as PtDGAT2A (Phatr2_49462), PtDGAT2B (Phatr2_49544), PtDGAT2C (Phatr2_31662) and PtDGAT2D (Phatr2_43469), have been characterised in TAG deficient yeast [19]. Of these only PtDGAT2B restored lipid body formation and showed a preferential substrate specificity for unsaturated FAs. Additionally, transcript analysis revealed that PtDGAT2A and PtDGAT2B were upregulated prior to the onset of TAG accumulation. Further studies indicated that the overexpression of PtDGAT2D in P. tricornutum resulted in an increased flux of photosynthetically fixed carbon towards lipids leading to a two-fold higher lipid content in transgenic cells compared to wild type (WT), alongside a reduction (15\%) in cell growth [21]. In contrast, overexpression of a chloroplast DGAT2 homolog from the diatom T. pseudonana, (Thaps3_25595), significantly increased TAG accumulation without a negative effect on growth or biomass accumulation [22].

Collectively, these studies illustrate how manipulation of DGAT genes in microalgae can increase their lipid content and modify fatty acid composition. Moreover, it is reasonable to suggest that they might 
participate in producing TAGs with enhanced levels of long-chain polyunsaturated fatty acids (LC-PUFAs) in microalgae (i.e. increasing incorporation of eicosapentaenoic acid, 20:5 $\Delta^{5,8,11,14,17}$, EPA and docosahexaenoic acid, 22:6 $4,7,10,13,16,19$, DHA. Recently we have engineered $P$. tricornutum to accumulate enhanced levels of DHA by overexpressing the $\Delta 5$-elongase from the picoalga 0 . tauri [23] and demonstrated the potential of this transgenic strain for industrial production of omega-3 LC-PUFAs in phototrophic and heterotrophic conditions $[24,25]$. The DHA content in transgenic Pt_OtElo5 was increased up to eight-fold that of WT levels and several novel LC-PUFA containing TAG species were detected. Further work has demonstrated the feasibility of multifunctionalizing the Pt_OtElo5 strain, combining the accumulation of high levels of EPA and DHA with recombinant protein production [26]. In the work presented here, we studied the effect of transgenic expression of the three most promising candidate DGAT2's, namely P. tricornutum DGAT2A, DGAT2B and T. pseudonana DGAT2 (TpDGAT2), on lipid accumulation and omega-3 LC-PUFA incorporation into TAG in WT and transgenic strains. The impact of these enzymes on lipid production in vivo was studied via lipidomic analysis. Our results demonstrate that overexpression of PtDGAT2B significantly enhances TAG production and alters FA composition during nitrogen-replete and nitrogen-limiting conditions. This study provides better insight into TAG biosynthesis in $P$. tricornutum and suggests a promising approach for tailoring oil composition in microalgae.

\section{Results}

\section{Generation of transgenic $P$. tricornutum strains overexpressing candidate DGAT2s.}

The native codon sequences for $P$. tricornutum DGAT2A, DGAT2B and a T. pseudonana DGAT2 (TpDGAT2), codon-optimized for expression in P. tricornutum, were cloned into the pPhOS2 vector [23] and the resulting constructs (Pt_DGAT2A, Pt_DGAT2B and Pt_TpDGAT2) were then transformed into $P$. tricornutum via biolistics (as described in [23]). Multiple zeocin-resistant colonies obtained for each construct were confirmed by PCR and then used to inoculate cultures for further screening by gas chromatography coupled to ion flame detection (GC-FID) analysis of fatty acid methyl esters (FAMEs). From this screening, we obtained multiple independent transgenic lines for each construct (Additional file 1: Table S1). The FA profiles and lipid content of selected independent overexpression lines were then analysed and compared to that of WT. As previously observed [23] the main FAs in total lipid extracts of WT and transgenic cells in decreasing order were C16:1, EPA, C16:0 and C14:0 (Fig. 1a). Transgenic lines overexpressing Pt_DGAT2B and Tp_DGAT2 demonstrated slightly elevated DHA levels.

To visualise the impact of transgene expression, a Nile Red fluorescence assay was used to evaluate the ability of the candidate DGAT2 overexpressing lines to accumulate enhanced levels of neutral lipids. Previously, analysis of transcript levels of the DGAT2 genes under standard growth conditions showed that the expression level of PtDGAT2A and PtDGAT2B progressively increased from day two to day four, peaked and then decreased to a low level in the following days [27]. Hence, transgenic strains overexpressing DGAT2s were cultivated for three days in standard F/2 medium to the early exponential (E) phase $\left(2.5 \times 10^{6}\right.$ cells). Nile Red staining of neutral lipids showed substantial differences between 
different DGAT2 clones and WT (Fig. 1b). All PtDGAT2B expressing clones showed a marked increase of TAG, while Pt_DGAT2A and Tp_DGAT2 cells did not accumulate elevated oil levels compared to WT cells.

To confirm gene transcription and elevated expression of DGAT2 genes, quantitative real-time PCR (qRTPCR) was carried out on selected transgenic lines. Based on the results of Nile Red staining, one of the most promising transgenic lines overexpressing each of the DGAT2 isoforms were characterized. Transgenic cultures were grown in F/2 medium and RNA extracted at the early $E$ phase. Subsequent qRTPCR analysis confirmed expression of Pt_DGAT2A_1, Pt_DGAT2B_5 and Tp_DGAT2_6 transcripts in the selected transgenic strains and demonstrated that the Pt_DGAT2B transgene is expressed at higher levels than the other two transgenes, Tp_DGAT2 and Pt_DGAT2A (Fig. 1c). Although the genes were under the control of the same promoter, the relative expression levels for the three DGAT2 isoforms were significantly different ( $\mathrm{p}<0.001$, for expression of both DGAT2A and of DGAT2B genes). For cell lines overexpressing native PtDGAT2A and PtDGAT2B, transcripts were increased relative to WT by 2.3 and 3.0fold, respectively. Based on the combined analysis of FA profiles, Nile Red staining and qRT-PCR analysis, the clone with the highest lipid content, Pt_DGAT2B_5, now designated DGAT2B, was taken forward for further detailed analysis.

\section{Generation of transgenic $P$. tricomutum strains co-expressing PtDGAT2B and $\triangle 5$-elongase from the picoalga 0 . tauri.}

To evaluate the effect of PtDGAT2B overexpression on the incorporation of omega-3 LC-PUFAs in TAG, coding sequences for the $O$. tauri $\triangle 5$-elongase (OtElo5) and PtDGAT2B were cloned in the previously described pPhOS2 vector [23], generating DGATElo construct. Ten individual zeocin-resistant clones were confirmed by PCR and screened for FA content (Additional file 2: Table S2). Four of the independent clones containing the highest levels of the new fatty acid, docosapentaenoic acid (DPA, 22:5n-3), the product of elongating activity of $\triangle 5$-elongase, and DHA were selected for further analysis. FAMEs analysis of the selected transgenic strains confirmed the presence of DPA in the range of 1.8-4.6\% accompanied by increased DHA levels (up to $3.8-8.5 \%$ compared to $1.8 \%$ in WT) in the stationary (S) phase of growth (Fig. 2a). Compared to WT, transgenic clones demonstrated substantially altered fatty acid profiles, containing on average 2-fold higher levels of $16: 3$ and 16:4, while the levels of C18:2 and C18:3n-3 were slightly reduced. The lipid content of these clones was further screened by Nile Red assay (Fig. 2b). The clone with the highest level of DHA and enhanced neutral lipid content, DGATElo_8 (designated DGATElo) was taken forward for further analysis.

\section{Cell growth and transgene expression: the impact of PtDGAT2B overexpression under N-replete and N- deplete conditions.}

Nutrient deprivation has been shown to induce TAG accumulation in microalgae [4]. To address the role of PtDGAT2B in TAG synthesis we studied cell growth under $\mathrm{N}$-replete and $\mathrm{N}$-deplete conditions during the most active phase of oil accumulation (72 hrs). N-replete F/2 media was supplemented with additional $P$ and $\mathrm{N}$ according to Abida et al., to ensure that these elements were not limiting during cell growth [28]. Ndeplete media contained F/2 enriched with added $\mathrm{P}$ and no $\mathrm{N}$. The growth rates and lipid accumulation of 
transgenic lines were determined under both $\mathrm{N}$-replete and $\mathrm{N}$-depleted conditions at 24-hour intervals for a total of 72 hours. The analysis of the growth profiles showed that there were no differences in growth patterns between the transgenic lines and the WT under both conditions. The rate of cell division under both $\mathrm{N}$-replete and $\mathrm{N}$-deplete conditions was both linear and consistent for transgenic and WT cells. (Fig. $3 a, b)$. Whereas the overall level of cell division differed substantially between the two nitrogen conditions $(p<0.01)$, decreasing in the N-deprived cultures. To confirm transcription of both the OtElo5 and PtDGAT2B genes during the experiment, RNA was extracted from triplicate cultures at $72 \mathrm{~h}$ of growth after resuspension in either $\mathrm{N}$-replete or $\mathrm{N}$-free media. Following cDNA synthesis qRT-PCR confirmed that overexpression of PtDGAT2B and transcription of the OtElo5 gene were occurring under experimental conditions (Fig. 3c, d). We have previously described the successful expression of OtElo5 under the endogenous $f c p A$ promoter of highly expressed fcpA gene [23]. However, $f c p$ promoters may not be strong enough to overexpress introduced activities due to the presence of light-responsive cis-regulatory elements [29] and stability under low nutrient conditions. Seo et al. showed that the endogenous elongation factor 2 (EF2) promoter isolated from $P$. tricornutum drove the expression of a transgene 1.2fold stronger than that driven by the $f c p$ promoter in light conditions and was stable throughout light and dark cycles [30]. However, recently we have shown that the expression of the $\Delta 5$-elongase (0tElo5) gene with an EF2 promoter resulted in comparable levels of EPA and DHA to that in transgenic strains in which the OtElo5 gene was under control of the $f c p A$ promoter [26]. In this study we tested both promotors' efficacies in response to Nitrogen treatment in transgenic lines expressing OtElo5 (Additional file 3: Table S3). The results indicate that the $f c p A$ promoter is suitable for transgene expression of DGAT2B in $P$. tricornutum when grown in different $\mathrm{N}$ conditions.

\section{Effect of PtDGAT2B overexpression on fatty acid composition under $\mathrm{N}$ - replete and $\mathrm{N}$-deplete conditions.}

To assess the impact of PtDGAT2B overexpression on cell FA profiles, cells were grown in $\mathrm{N}$ - replete and $\mathrm{N}$-deplete conditions and analysed at 24,48 and $72 \mathrm{~h}$ time points respectively. The fatty acid profiles of selected independent overexpression lines DGAT2B and DGATElo were then compared to that of WT and transgenic Pt_OtElo5. Fatty acid composition (Mol\%) was clearly affected by $\mathrm{N}$ treatment, for example DGAT2B cells accumulated slightly higher levels of DHA in comparison to WT in N-replete conditions (Fig. 4). Irrespective of $\mathrm{N}$ treatment, the highest DHA levels were observed in DGATElo cells. As expected, cells expressing OtElo5 (Pt_OtElo5 and DGATElo) contained decreased EPA compared to that of DGAT2B and WT at all time points due to the action of the $\triangle 5$-elongase (Fig. 4). The novel FA DPA was only present in the strains expressing OtElo5 (Pt_OtElo5 and DGATElo).

When total FAMEs were quantified (expressed as nmol mg-1), the main differences between strains were observed in the content of 16:0 and 16:1 (Additional file 4: Figure S1). As expected, [28, 31], $\mathrm{N}$ deprivation was accompanied by significant changes in the levels of 16:0 and 16:1 $(p<0.001)$ and time $(p<0.001)$. The abundance of 16:0 and 16:1 was higher in N-starved cultures and increased over time $(p=0.002$ and $p$ $=0.019$ respectively) in all strains. Higher levels of $16: 0$ and $16: 1$ were observed in cells overexpressing PtDGAT2B ( $\mathrm{p}=0.003$ and $\mathrm{p}<0.01$ respectively), where the content of $16: 0$ and $16: 1$ in $\mathrm{N}$-limited conditions at $72 \mathrm{~h}$ reached $442 \mathrm{nmol} . \mathrm{mg}^{-1}$ and $443 \mathrm{nmol} . \mathrm{mg}^{-1}$ respectively, corresponding to a 2- and 1.5 -fold 
increase in comparison to the WT. Overall, the accumulation of LC-PUFA (EPA, DPA and DHA) was largely unaffected either by $\mathrm{N}$-depletion or time. However, in all experimental conditions, transgenic cells accumulated higher levels of DHA than WT, with most efficient accumulation observed in DGATElo. Interestingly, after 72 hours in N-depleted medium, DGATElo cells accumulated 2.8- fold higher levels of EPA and 2-fold higher levels of DPA than the single Pt_OtElo5 strain $\left(73 \mathrm{nmol} . \mathrm{mg}^{-1}\right.$ and $26 \mathrm{nmol} . \mathrm{mg}^{-1}$ respectively). These results indicate that PtDGAT2B plays an important role in FA accumulation under $\mathrm{N}$ replete and N-deplete conditions. Although PtDGAT2B has a strong substrate preference for $\mathrm{C} 16 \mathrm{FAs}$, it also demonstrates the broad substrate specificity of this acyltransferase, particularly towards LC-PUFAs, such as EPA, DPA and DHA.

\section{Lipidomic analysis of WT and transgenic cells under N-replete and N-deplete conditions}

To gain insight into the ability of PtDGAT2B to enhance TAG accumulation we performed a comprehensive analysis comparing glycerolipid profiles at different time points for WT and transgenic strains grown in N-replete and N-deprived conditions. Mass spectrometry (ESI-MS/MS) based lipidomic approaches have been used previously in our laboratory to characterise $P$. tricornutum lipid turnover and remodelling. The reliability of the analysis was demonstrated by the principle component analysis (PCA), which showed clear clustering of the strain and treatment replicates (Fig. 5). Cells were harvested for analysis at 24, 48 and 72 hours, corresponding to $E$ and $S$ phases of growth. Furthermore, the sampling period captured the impact of transgene expression and the application of nitrogen stress, which induces a significant reorganisation of cellular lipid metabolism. A multivariate statistical approach (PCA) was used to decrypt the significant differences observed in such a comprehensive analysis. The first two principal components account for $48.69 \%$ of variation within the data and are shown in Figure 5 . Clear patterns are visible in this 2-dimensional representation of the data, with the first principal component separating out $\mathrm{N}$ - and $\mathrm{N}+$ treatments and within the $\mathrm{N}$ - treatments distinguishing between the different time effects. There is separation of the data set over time under $\mathrm{N}$-deplete conditions, but not $\mathrm{N}$-replete, demonstrating the accumulation and remodelling of lipids in response to $\mathrm{N}$ deprivation. The second principal component shows separation of the different transgenic lines, with Pt_OtElo5 and DGATElo clustering together and DGAT2B and WT separating into a second group.

The relative contribution each lipid has on the direction of each principle component was determined. Additional file 5: Table S4 lists the 15 lipids with highest loadings associated with these dimensions. Under N-deplete conditions, the first principal component (PC-1), a high TAG and low polar lipid response is clear. Specific TAG species with high abundance are 48:2 and 48:3 presumptively 16:0, 16:1- containing TAGs and the EPA- containing TAG 50:3. Other lipid species contributing to this dimension are the galactolipids mono- and digalactosyldiacylglycerol (MGDG and DGDG). The second principal component (PC-2) can be interpreted as an average of selected lipids, namely phosphatidylcholines (PC), Lyso-PCs (LPC) and diacylglycerylhydroxymethyltrimethyl- $\beta$-alanine (DGTA), thus high values of PC-2 (associated with WT and DGAT strains) correspond to high abundance in these lipids. Compared to WT and DGAT2B, cells expressing the OtElo5 gene contain lipids (LPC, PC and DGTA) abundant in DPA and DHA. Several important observations can be made from the PCA analysis. When considering the full set of lipid 
species, cells overexpressing the PtDGAT2B gene cluster with WT although the lipid content is altered (Fig. 6, Additional file 4: Figure S1 and Additional File 6: Figure S2). Introduction of the OtElo5 gene separates out cell lines expressing it under $\mathrm{N}$-replete and $\mathrm{N}$-deplete conditions. Cells expressing the OtElo5 gene and overexpressing PtDGAT2B, individually or in combination, cluster together. These differences between the cell lines are driven by lipids containing DPA and DHA, e.g. DGTA 44:12 among others. As expected, the PCA plots show that the biggest impact on distribution is $\mathrm{N}$ stress and that this is driven by changes in abundance of TAG. Other lipid species contribute to changes in lipid profile, but they are largely minor components. These data suggest that in response to $\mathrm{N}$ stress, de novo biosynthesis of TAG primarily utilizing the ER-localized Kennedy pathway, rather than remodelling of membrane lipids, is the major driver in TAG accumulation.

\section{Impact of transgene expression on TAG accumulation under $\mathrm{N}$-replete and $\mathrm{N}$-deplete conditions}

First, we examined the impact of PtDGAT2B overexpression on lipid accumulation in transgenic cells. Both the DGAT2B and DGATElo cells contained elevated TAG compared to WT and Pt_OtElo5 cells irrespective of $\mathrm{N}$ conditions at all-time points (Fig. 6 and Additional file 7: Table S5). The results demonstrated that $\mathrm{N}$ deprivation is not required for enhanced TAG accumulation in the PtDGAT2B overexpressing strains. Under N-replete conditions at $72 \mathrm{~h}$ the DGAT2B cells accumulated $22 \mathrm{nmol} . \mathrm{mg}^{-1}$ DW of TAG, which corresponded to a 3.5-fold increase in comparison to the WT. These results indicated that DGAT2B overexpression markedly improved lipid productivity in cells of $P$. tricornutum. Since $N$ deprivation stimulates lipid production in various microalgae species, $P$. tricornutum WT and transgenic lines were grown and analysed in N-replete and N-deplete conditions. $\mathrm{N}$ depletion induced TAG accumulation in all cell types and resulted in substantially higher TAG than under replete conditions. We observed a 2-fold increase in TAGs in comparison to the control in the DGATElo cells (with the highest level of $313.7 \mathrm{nmol} . \mathrm{mg}^{-1}$ ) and a 1.4-fold increase in DGAT2B and Pt_Elo5 cells (240 nmol. $\mathrm{mg}^{-1}$ and 243 nmol.mg ${ }^{-1}$ respectively) after $72 \mathrm{~h}$.

To further examine lipid production in P. tricornutum cells, TAG accumulation in lipid bodies of the WT and transgenic cells was assessed qualitatively by BODIPY 505/515 staining and confocal microscopy (Additional file 6: Figure S2). Under N-replete conditions oil bodies in WT and Pt_OtElo5 cells remain almost unchanged over the time course, whereas PtDGAT2B overexpressing cells contained larger oil bodies at each time point. Neutral lipid content in all cell types increased considerably in N-starved cultures compared to $\mathrm{N}$-replete conditions. DGAT2B and DGATElo cells contained larger and more numerous oil bodies relatively to WT and Pt_OtElo5 over the time. The increase and volume of oil bodies in $P$. tricornutum cells expressing DGAT2B and/or OtElo5 was consistent with the increase in neutral lipid content measured by mass spectrometry. To detect the impact of transgene expression on FA assembly in TAG further detailed analysis by ESI-MS/MS was undertaken. FA content in TAG showed that along with the different levels of TAG accumulation between strains, there were also differences in TAG FA profiles and molecular species composition under $\mathrm{N}$-replete and $\mathrm{N}$-deplete conditions. DGATElo contained a significantly higher proportion of DHA in TAG than other strains under both conditions $(p=0.0003)$ (Fig 7 
a, b). However, DHA levels were slightly decreased in all cell types under $\mathrm{N}$ starvation in agreement with previous observations that LC-PUFAs levels decrease in N-depleted medium [32].

Also, in agreement with previous reports $[28,31]$ the most abundant TAG species under standard conditions in P. tricornutum cells were C16 containing species, 48:1, 48:2 and 48:3. A similar pattern was observed in all transgenic cells. However, the levels of C16 FA-containing species and those consisting of a mix of C16-C18 FA (50:2 and 50:3) in DGAT2B cells increased 3-8 fold compared to WT, thus demonstrating enhanced channelling of C16 FA into TAG in the presence of PtDGAT2B. Elevated 54:6, 54:7 and 54:8 corresponding to DHA/DPA-containing TAG species, were detected in DGATElo cells (Additional file 8: Figure S3). The levels of TAG species containing C16 FA were dramatically changed with nitrogen treatment. Under N-deplete conditions levels of 48:1, 48:2 and 48:3 TAG were substantially increased in all cell types. DGAT2B and DGATElo cells contained elevated levels of 50:2 and 50:3 TAG. DHA-containing TAG species showed differing responses to N depletion. Notably, DHA TAG 54:7 increased up to $64 \%$ in DGATElo, whereas $54: 8$ and $54: 6$ increased up to $39 \%$ and $8.7 \%$ respectively. Minor new DHA-containing species, e.g. 56:8, 56:9, 56:10 and 56:11, were mainly observed in cells grown in N-deplete media (Additional file 8: Figure S3). The data collectively demonstrated that the broad substrate specificity associated with DGAT2B facilitated a pathway for DHA synthesis and incorporation into TAG as lipid droplets. Moreover, the combined expression of DGAT2B and OtElo5 may potentially increase lipid production without altering cellular growth.

\section{Polar lipid accumulation in WT and transgenic cells under $\mathrm{N}$-replete and $\mathrm{N}$-deplete conditions}

To better understand the impact of transgene expression on the lipidome of DGAT2B overexpressing cells and determine if the substantial changes in TAG composition under $\mathrm{N}$ stress are a result principally of de novo biosynthesis of TAG, as indicated by multivariate analysis, or by lipid remodelling, the polar lipid content of WT and transgenic cells was examined using ESI-MS/MS approaches. The structural reorganisation of chloroplast membranes in response to $\mathrm{N}$ depletion and recycling of membrane lipids into TAG has been reported in many microalgae strains. Nitrogen depletion is often accompanied by the movement of LC-PUFA from MGDG and DGDG to TAG. Assessment of the polar lipid classes illustrates a profile that was similar to that of the $P$. tricornutum Pt1 ecotype reported by Abida et al. [28] and dominated by MGDG, PC, phosphatidylglycerol (PG) and DGDG under both $\mathrm{N}$ conditions (Additional file 9: Figure S4). The primary impact of $\mathrm{N}$ depletion on polar lipids in all strains was a reduction in all classes. Reviewing the individual polar lipid molecular species under N-replete conditions (Additional file 10; Figure S5), the most abundant species in MGDG of all cell types is 36:8, likely comprising of 20:5 and 16:3. Molecular species found in MGDG of DGAT2B cells show similar profiles to that of WT. Transgenic clones expressing the OtElo5 gene, have distinct MGDG profiles from that of WT and DGAT2B strains and are characterized by enhanced levels of $32: 5$ and 32:6 (a mix of 16:2 and 16:3) and lower levels of 36:8, correlating with a decrease of EPA due to OtElo5 activity in these transformants and consequently, reduced import of EPA into the chloroplast via a putative omega pathway [30] (Additional file 10: Figure S5). Under N-deplete conditions there was an overall reduction in EPA-containing 36:6, 36:8, 36:9 MGDG species in all cell types correlating with a general decrease in EPA content. MGDG 32:5 and 32:6 were 
also reduced considerably under $\mathrm{N}$ depletion. An assessment of other galactolipid species showed differing responses in both DGDG and sulfoquinovosyldiacylglycerol (SQDG) (Additional file 10: Figure S5). Consistent with previous observations [28], 36:7 and 36:6 species were present at high levels in WT and are likely to contain $20: 5$ and $16: 2 / 16: 1$. All transgenic clones contain molecular species similar to that of WT indicating that transgene expression did not have a significant impact on DGDG and SQDG. The profile of SQDG in all cells was dominated by 30:1 (14:0/16:1) and 32:1 (16:1/16:0) under N-replete conditions and showed minor decrease under $\mathrm{N}$-deprived conditions. The main EPA-containing species of SQDG, 36.5 (20:5 and 16:0), were present only in WT and DGAT2B cells, correlating with reduced amounts of EPA in OtElo5-expressing clones. In contrast to Popko et al. [31] a lower proportion of a betaine glycerolipid has been observed (Additional file 11: Figure S6). This may be due to the different culture growth conditions used in both experiments.

Under N-replete conditions the dominant PC species in WT and DGAT2B were EPA-containing 36:5, 36:6, 38:7 and 40:10 (Additional file 12; Figure 7 a \& b). The proportions of these species remained mostly unchanged under both $\mathrm{N}$ conditions. The PC pool of OtElo5 expressing strains in N-replete medium contained highly elevated levels of species incorporating newly-synthesized DPA and DHA. The major species in these transformants were those, consisting of the mix of LC-PUFAs and C16 FA: 38:5 (likely 22:5 and 16:0), 38:6 and 38:7 (a mix of 22:6 and C16:0/C16:1), and those with a mix of EPA, DPA and DHA, such as 40:7; 42:9, 42:10 and 42:11. Four new PC molecular species 42:8, 44:10, 44:11 and 44:12 likely containing DHA and DPA were detected in transgenic clones expressing OtElo5. An increase in DHAand DPA-containing species suggests that the synthesis of these LC-PUFAs takes place in the PC pool. Species containing EPA (36:5 and 40:10) in OtElo5 expressers were markedly reduced, likely corresponding to changes in EPA levels. Interestingly, under N-limited conditions, levels of DHA containing species, 42:10 and 42:11, increased in DGATElo cells.

Analysis of PG showed a consistent predominance of C16-consisting 32:1 and 32:2 species, and EPAcontaining $36: 5$ and $36: 6$ species in all the transgenic clones and WT under both N-conditions. Pt_OtElo5 cells contained higher 32:1 in PG compared to WT cells grown in replete and N-deprived medium, the significance of this is not clear. The measured amount of PG reduced in all cell types under N-deplete conditions (Additional file 12; Figure 7c \& d). Notably, phosphatidylinositol (PI) of all cell types comprised mainly $16: 1 / 16: 0$ and was stable with $N$ treatment, indicating that this lipid pool was not remodelled in response to $\mathrm{N}$ deprivation (Additional file 12; Figure 7 e \& f). Phosphatidylethanolamine (PE) profiles were very different between strains grown under $\mathrm{N}$-replete and $\mathrm{N}$-deplete conditions. In $\mathrm{N}$-replete medium PE of WT and DGAT2B cells contained mainly 40:10 (20:5 and 20:5) and 42:11 (20:5 and 22:6) molecular species. New 42:10 (20:5 and 22:5) and enhanced levels of 42:11 species were detected in Pt_OtElo5 and DGATElo transgenic cells (Additional file 12; Figure $7 \mathrm{~g} \& \mathrm{~h}$ ). The presence of these new species can be attributed to expression of the OtElo5 gene as they are absent in WT and DGAT2B cells. Jointly the data for PI and PE indicates that they do not contribute significantly to the biosynthesis of TAG in response to $\mathrm{N}$ deprivation. 
To complete our analysis, ESI-MS/MS profiling was used to determine the FA composition of lyso-PC in WT and transgenic cells under the different $N$ treatments (Fig. 8). The major FAs in WT and DGAT2B cells grown under both conditions were 18:2, EPA and DHA, whereas in Pt_OtElo5 and DGATElo transgenic cells FA profiles were dominated by DPA and DHA, the products of $\triangle 5$-elongation of EPA and subsequent $\Delta 4$-desaturation. In addition, two new FAs, 22:4 (the product of 20:4n-6 $\Delta 5$-elongation) and 24:6 (probably the product of 22:6 elongation) were observed in Pt_OtElo5 and DGATElo transformants. There was a 2fold increase in DHA-containing lyso-PC in Pt_OtElo5 compared to WT and DGAT2B and a 5-fold increase in DGATElo in N-deplete conditions (Fig. 8b). The rise in DHA levels correlated with a reduction of 16:0, $18: 1,18: 2,18: 3$ and EPA in the OtElo5 expressing strains. The levels of DHA-containing lyso-PC were 2fold higher in DGATElo compared to that of OtElo5 cells. Overexpression of the DGAT2B gene on its own has only small impact on the DHA levels in N-replete cultures. These data indicate a crucial role lyso-PC in LC-PUFA synthesis and incorporation into complex lipid species.

\section{Discussion}

DGATs are thought to be rate-limiting enzymes and are considered the main contributors to oil biosynthesis in microalgae. Most microalgal species, including $P$. tricornutum have multiple copies of DGAT2 genes. Hence, identification of the best candidates for enhancing LC-PUFA production and TAG accumulation represents a promising approach to the metabolic engineering of strains with desired qualities. However, a functional role for the multiple type 2 DGATs found in algal species remains unclear. In microalgae $\mathrm{N}$ stress triggers TAG biosynthesis and often induces DGAT expression [6]. In $P$. tricornutum during N-replete and N-deplete conditions, TAGs and the precursor DAG are mainly composed of 16:0 and $16: 1$, whereas EPA and DHA are minor species $[31,28]$. This suggests that LC-PUFAs are likely transferred to TAGs by either one of the four DGAT2s identified in P. tricornutum [27] via the Kennedy pathway or a route involving phosphatidyl diacylglycerol acyltransferase PDAT [2]. We investigated the impact of the overexpression of DGAT2 isoforms in P. tricornutum WT and Pt_OtElo5 strains on overall TAG accumulation and specifically DHA partitioning in TAG. To achieve this, transgenic cell lines overexpressing the three most promising candidate DGAT2s, PtDGAT2A, PtDGAT2B and TpDGAT2, were generated and analysed for their fatty acid composition, relative overexpression levels and lipid content. Transgenic cells overexpressing PtDGAT2B showed the highest mRNA levels (a 3-fold increase when normalized to WT strain), elevated lipid content evaluated via a Nile Red fluorescence assay (Fig. 1) and increased proportions of DHA in total FAs (Fig. 2). To further improve LC-PUFA content and partitioning in TAG we coexpressed PtDGAT2B, the most efficient among the three studied DGAT2B candidates in enhancing neutral lipids levels, with the heterologous $O$. tauri $\triangle 5$-elongase (OtElo5) and analysed the FA profiles and lipid production in cells grown in N-replete and $\mathrm{N}$-deplete medium.

FAMEs analysis indicated that PtDGAT2B can significantly alter FA composition in transgenic clones under $\mathrm{N}$-replete and $\mathrm{N}$-deplete conditions. The main differences between strains were observed in the levels of 16:0, 16:1, EPA, DPA and DHA (Fig. 3, Additional file 1: Table 1). In clones overexpressing PtDGAT2B 16:0 and 16:1 content increased 5- and 4-fold respectively at $72 \mathrm{~h}$ in $\mathrm{N}$-deprived cultures and was considerably higher than that in WT at each time point. Interestingly, DHA levels in strains 
overexpressing PtDGAT2B were higher than that in other strains and not affected by nitrogen depletion. Overexpression of PtDGAT2B resulted in significant increases in TAG content in transgenic cells under $\mathrm{N}$ replete and $\mathrm{N}$-deplete conditions at all-time points (Fig. 7 and Additional file 5 Table S4). A 4-fold increase in TAG accumulation observed in DGAT2B cells under N-replete conditions demonstrates that the requirement for nutrient depletion to increase lipid accumulation could be overcome by using PtDGAT2B as a tool for manipulating TAG content. Expression of the transgenes did not significantly alter cell growth rate in the different cell lines (Fig. 3). This contrasts with a previous study in which a PtDGAT2D gene was overexpressed in P. tricornutum; cells contained elevated TAG but grew $15 \%$ slower than WT [21].

From a biotechnology perspective, the ability to engineer strains that overproduce TAG under normal growth conditions is highly desirable as nutrient depletion negatively affects cell growth. Overexpression of another type 2 DGAT, NoDGTT5, in N. oceanica CCMP1779 grown in N-replete medium resulted in increased TAG synthesis normally observed only after $\mathrm{N}$ deprivation [15]. However, lipid accumulation was accompanied by strongly reduced growth rates normally observed under $\mathrm{N}$-deplete conditions.

$\mathrm{N}$ starvation triggers substantial TAG accumulation in all cell types with the highest 37 -fold increase in DGATElo cells when compared with N-replete conditions and 1.8-fold higher level of TAG than in the WT in $\mathrm{N}$-deplete medium. Under $\mathrm{N}$ stress TAG levels in DGAT2B transformants increased 11-fold when compared with N-replete conditions and $~ 1.5$-fold compared with these in WT cells (Fig. 7, Additional file 5: Table S4). Recent studies of DGAT isoforms demonstrated that in P. tricornutum only PtDGAT1 was strongly regulated by nitrogen depletion [14], whereas PtDGAT2B was highly expressed under N-replete conditions before the onset of TAG accumulation [27]. Our findings suggest that PtDGAT2B might contribute to TAG accumulation under both $\mathrm{N}$-replete and $\mathrm{N}$-deplete conditions since we observed significant increase in TAG content in transgenic cells overexpressing PtDGAT2B irrespective of $\mathrm{N}$ treatment. We also demonstrated that the $f c p$ promoter is suitable for gene overexpression under $\mathrm{N}$ deprivation (Fig 3.; Additional file 3: Table S3). This contrasts with the results of expression of a NoDGTT5 under the control of EF promoter in N. oceanica in which elevated TAG was observed under Nreplete, but not deplete conditions. However, the use of the native gene promoter did increase TAG biosynthesis under deplete conditions [15].

Lipidomic analysis demonstrated that PtDGAT2B possesses broad substrate specificity and can incorporate different acyl-CoA species, including C16 and LC-PUFAs. Overexpression of DGAT2B resulted in an increase in TAGs containing C16 species, whereas cells expressing OtElo5 accumulated elevated levels of LC-PUFA containing TAG (Fig. 7). The levels of DHA-containing TAG species were higher in cells coexpressing both OtElo5 and DGAT2B indicating that PtDGAT2B enhances the incorporation and transfer of endogenous C16:0 and C16:1 fatty acids and newly synthesized DPA and DHA into TAG.

Changes in membrane lipids were principally observed in MGDG, DGDG and PG reflecting the rapid senescence of chloroplast membranes, whilst total amounts of PC, DGTA and lyso-PC did not change significantly over the time in all strains. Notably there were significant reductions in EPA- and C16- 
containing MGDG species. Reductions in C16- and EPA-containing PC and DGTA also correlated with an increase in TAG suggesting that these species are entering TAG biosynthesis, possibly via the dynamic lyso-PC pool (Fig. 8). PDAT with broad substrate specificity have been shown to be a major contributor to TAG biosynthesis in $C$. reinhardtii under N-deplete conditions [34]. Up-regulation of PDAT and enzymes associated with TAG biosynthesis under $\mathrm{N}$ stress have also been reported in P. tricornutum [35]. However, the pool size of polar lipids is not sufficient to account for the increase in TAG indicating that whilst lipid remodelling occurs under N-deplete conditions, de novo TAG biosynthesis is the major driver behind TAG accumulation. Synthesis of TAG requires a carbon source and the decline in photosynthetic capacity (illustrated here by the reduction in galactolipids) with $\mathrm{N}$ depletion, indicates that the source of this carbon must be from the remaining photosynthetic capacity and stored carbohydrates. This observation is supported by the multivariate PCA analysis (Fig. 6) and has been reported previously by Abida et al. in the Pt1 ecotype of $P$. tricornutum [28] in contrast to a previous report which proposed betaine lipids as major contributors to TAG formation [31]. An effect that is likely due to differences in growth conditions.

\section{Conclusions}

The overall goal of this work was to evaluate the functional role of PtDGAT2B in the synthesis and assembly of TAG. In this study we have successfully demonstrated that PtDGAT2B is an important contributor to TAG biosynthesis and a useful tool to produce high accumulating lipid strains for industrial biotechnology. By overexpressing PtDGATB we have successfully shown how an engineered transgenic strain of $P$. tricornutum can accumulate elevated TAG levels under $\mathrm{N}$-replete growth conditions. Moreover, the application of a multigene approach, co-expressing DGAT2B with the heterologous $\Delta 5$-elongase from the picoalga $O$. tauri, produced a significant increase in DHA containing TAG, confirming the broad substrate specificity of this DGAT. Furthermore, heterologous expression of transgenes did not impact cell growth rates demonstrating the advantage of this approach to engineer improved lipid content and composition. DGATElo cells represent a promising source of DHA that contain enhanced TAG levels without the requirement for $\mathrm{N}$ depletion. Although nitrogen stress is usually used to enhance lipid productivity, this study provides evidence that it is possible to increase TAG content without nutrient stress and engineer TAG to contain highly desirable FAs such as DHA and DPA. To match the level of TAG accumulation under $\mathrm{N}$ depletion without effecting the cell growth further knowledge of regulatory mechanisms of lipid production is required.

\section{Methods}

\section{Strains and growth conditions}

Phaeodactylum tricornutum ecotype Pt4 was grown in $\mathrm{F} / 2$ media [32], at $20^{\circ} \mathrm{C}$ with moderate shaking and constant illumination $\left(60 \mu \mathrm{mol}\right.$ photons. $\left.\mathrm{m}^{-2} \cdot \mathrm{s}^{-1}\right)$. For $\mathrm{N}$ stress experiments, replete $\mathrm{F} / 2$ media contained enriched phosphate $(P)$ and nitrogen $(N)$ and enriched $P$ with no $N$ in deplete conditions as described by Abida et al. [28]. Cells were grown in enriched F/2 media to the log phase of growth. Cells were then pelleted by centrifugation and used to inoculate $50 \mathrm{ml}$ cultures of replete or depleted media at 3 
$x 10^{6}$ cells $/ \mathrm{ml}$. For each strain $n=4-9$ depending on the cell type. Cells were counted at time points of $24 \mathrm{~h}$, $48 \mathrm{~h}$ and $72 \mathrm{~h}$ at which point they were harvested by centrifugation, pellets were washed with $3 \%$ ammonium formate, pelleted again, snap frozen in liquid nitrogen and freeze dried.

\section{Plasmid design and cloning}

Plasmids were constructed containing either a single PtDGAT2B gene or two-gene cassette with PtDGAT2B and OtElo5 genes. The coding sequences for PtDGAT2A, PtDGAT2B and TpDGAT2 were inserted as $\mathrm{Kpnl}$-Sacl fragments into position 1 in pPHOS vector under the control of either $f c p A$ or EF2 promoter using a multigene vector described previously [23]. The efficacy of the fcpA promoter in $\mathrm{N}$ depleted and $\mathrm{N}$-replete conditions was tested and compared to the EF2 promoter (Supplementary Table S3). The codon-optimised for expression in P. tricornutum coding sequence for $O$. tauri OtElo5 was inserted as BamHI-Xbal fragment into position 2 of pPHOS to generate double-gene construct DGATElo. Constructs were transformed into $P$. tricornutum via biolistic according to methods previously described [36]. Positive transformants were selected using the ble marker and confirmed by PCR.

\section{Fatty acid analysis}

Total FA content of cells was measured by gas chromatography flame ionisation detection (GC-FID) as described previously [23]. Analysis was carried out using Agilent Chemstation software. Total FAMEs were quantified using 17:0 fatty acid standard (Sigma).

\section{Nile Red staining of non-polar lipids}

Non-polar lipids and oil droplets were measured using Nile Red (Sigma-Aldrich) fluorescence staining (excitation at $485-512 \mathrm{~nm}$ and emission at $590-610 \mathrm{~nm}$ ) using a fluorescent microplate reader (Fluostar Omega, BMG Labtech) as described previously [37]. $200 \mu$ culture containing $2.5 \times 10^{6}$ cells in the exponential phase of growth was stained with $50 \mu \mathrm{l}$ Nile Red stock $\left(2.5 \mu \mathrm{g} \cdot \mathrm{ml}^{-1}\right.$ in $25 \%$ DMSO). Blank measurements (cells incubated without Nile Red stain) were subtracted from test samples and total fluorescence was expressed by dividing fluorescence over that of WT cells.

\section{RNA isolation and qRT-PCR conditions}

Total RNA was extracted from $10^{8}$ cells using an RNeasy Plant mini kit (Qiagen, Germany) according to the manufacturer's instructions, with minor modifications. Breakage of the algal cells was aided by freezing in liquid nitrogen prior to the addition of the Qiagen kit RLT lysis buffer. The cell mixture was then sonicated for $1 \mathrm{~min}$ followed by an incubation on ice for $1 \mathrm{~min}$, this process was repeated twice. RNasefree DNase (Promega, USA) was used to remove traces of genomic DNA and the RNA was subsequently purified using RNeasy mini kit columns (Qiagen). The concentration of the extracted RNA was determined using a spectrophotometer at $260 \mathrm{~nm}$ and $0.5 \mu \mathrm{g}$ was reverse transcribed into cDNA using the Transcriptor First Strand cDNA synthesis kit (Roche Applied Science, Germany) according to the manufacturer's instructions. RPS, encoding the ribosomal small subunit $30 \mathrm{~S}$, was used as the 
housekeeping gene since the expression level of RPS has previously been shown to be stable in different conditions [38].

All remaining genes and the corresponding primer pairs used, designed with the Primer3Plus program, are listed in Additional file 13: Table S6. Relative gene expression levels were analysed using the $2^{-} \Delta \Delta C_{T}$ method using a LightCycler® 96 System (Roche Applied Science). Reactions were started by adding 20 ng cDNA to a mixture containing 1 x SYBR Green PCR Master Mix (Roche Applied Science) and $300 \mathrm{nM}$ of the specific primers in a total volume of $10 \mu \mathrm{l}$. The thermal-cycling conditions were as follows: $95^{\circ} \mathrm{C}$ for 10 min and 40 cycles at $95^{\circ} \mathrm{C}$ for $10 \mathrm{~s}, 60^{\circ} \mathrm{C}$ for $15 \mathrm{~s}$ and $15 \mathrm{~s}$ at $72^{\circ} \mathrm{C}$. Melting-curve analysis was performed to check for primer-dimer artifacts. Primer efficiencies were estimated by performing cDNA serial dilutions using the slope calculation supplied with the LightCycler ${ }^{\circledR} 96$ System Software (Roche Applied Science). For each gene, 3 independent biological replicates and two technical replicates were performed.

\section{Neutral lipid staining and visualisation by confocal microscopy}

Non-polar lipids and oil droplets were visualised using Bodipy staining and confocal microscopy according to Govender et al. [39]. Cells were grown in nitrogen replete or deplete conditions and non-polar lipids and neutral lipids were stained after $24 \mathrm{~h}, 48 \mathrm{~h}$ and $72 \mathrm{~h}$ with BODIPY. $0.5 \mathrm{ml}$ cells were pelleted at low speed and re-suspended in artificial sea water (Sigma) containing $1 \mu \mathrm{g} / \mathrm{ml}$ BODIPY (diluted from a stock of $1 \mathrm{mg} / \mathrm{ml}$ in DMSO). Cells were incubated for 10 minutes, pelleted by centrifugation and washed in sea water. Cells were then re-suspended in $15 \mu$ l sea water prior to confocal microscopy (Zeiss LSM 780).

\section{Quantitative lipid analysis}

Total lipids were extracted according to Abida et al., with some modifications [28]. $2 \mathrm{mg}$ freeze dried cells were re-suspended in $4 \mathrm{ml}$ propan-2-ol and heated at $80^{\circ} \mathrm{C}$ for 10 minutes. The solvent was transferred to a $50 \mathrm{ml}$ stoppered conical flask. The pellet was then extracted by grinding with a glass homogeniser in 2 $\mathrm{ml}$ methanol followed by $8 \mathrm{ml}$ chloroform. Flasks containing solvent extract were flushed with nitrogen gas and stirred for $1 \mathrm{~h}$ at room temperature. Samples were filtered through glass wool which was then washed with $3 \mathrm{ml}$ chloroform:methanol (2:1). $5 \mathrm{ml}$ 1\% (w/v) NaCl was added to initiate biphase formation and the chloroform phase dried under nitrogen gas. Total lipid extracts were resuspended in chloroform:methanol:ammonium acetate (1:2.2:0.1), filtered (0.45 $\mu \mathrm{m}$ Millex-FH filters, Merck Millipore, Germany), dried under a stream of nitrogen, flushed with nitrogen, and stored at $-80^{\circ} \mathrm{C}$. Quantitative analyses of TAG and polar lipids, which comprise phospholipids (PC, DGTA, PE, PI, PG, LPC) and galactolipids (DGDG, MGDG and SQDG), were carried out using electrospray ionization tandem triplequadrupole mass spectrometry (API 4000 QTRAP, SCIEX; ESI-MS/MS). The lipid extracts were infused at $15 \mu \mathrm{L} /$ min with an autosampler (HTS-xt PAL, CTC-PAL Analytics AG, Switzerland). Data acquisition and acyl group identification of the polar lipids was as described in Ruiz-Lopez et al. [40]. DGTA analyses were carried out in positive ion mode by scanning for precursors of $\mathrm{m} / z 236$. The internal standards for 
polar lipids were supplied by Avanti (Alabaster, AL, USA), incorporated as $0.857 \mathrm{nmol}$ of 13:0-LPC, 0.086 $\mathrm{nmol}$ of di24:1-PC, $0.080 \mathrm{nmol}$ of di14:0-PE, $0.800 \mathrm{nmol}$ of di18:0-PI, and $0.080 \mathrm{nmol}$ of di14:0-PG. The standards dissolved in chloroform and $25 \mu \mathrm{L}$ of the samples in chloroform were combined with chloroform/methanol/300 mM ammonium acetate (300:665:3.5 v/v) to make a final volume of $1 \mathrm{~mL}$. For quantifying TAG, $15 \mu \mathrm{L}$ of lipid extract and $0.857 \mathrm{nmol}$ of tri15:0-TAG (Nu-Chek Prep, Elysian, MN, USA) were combined with chloroform/methanol/300 mM ammonium acetate (24:24:1.75: $\mathrm{v} / \mathrm{v})$, to final a volume of $1 \mathrm{~mL}$ for direct infusion into the mass spectrometer. TAG was detected as $\left[\mathrm{M}+\mathrm{NH}_{4}\right]^{+}$ions by a series of different neutral loss scans, targeting losses of fatty acids. The data were processed using the program Lipid View Software (AB-Sciex, Framingham, MA, USA) where isotope corrections are applied. The peak area of each lipid was normalized to the internal standard and further normalized to the weight of the initial sample. There is variation in ionization efficiency among acyl glycerol species with different fatty acyl groups, and no response factors for individual species were determined in this study; therefore, the values are not directly proportional to the TAG contents of each species. However, the approach does allow a realistic comparison of TAG species across samples in this study.

\section{Statistical analysis}

Principal component analysis (PCA) was done on the complete dataset of 215 lipids in 138 treatment replicates (24 treatment combinations). Data were log transformed after the addition of a small offset calculated as half the minimum non-zero value. PCA was calculated using the correlation matrix, to give equal importance to each variable. The first two principal components account for $48.69 \%(27.95 \%$ and $20.76 \%$ ) of variation within the data and have clear geometric interpretation. The variation accounted for in the third and fourth roots were 10.17 and 4.93 respectively with little additional interpretation available for these dimensions. Analyses were performed in GenStat $17^{\text {th }}$ Edition and R 3.2.2.

\section{Abbreviations}

DAG - diacylglycerol; DGAT- diacylglycerol acyltransferase; DGDG, digalactosyldiacylglycerol; DGTA, diacylglycerylhydroxymethyltrimethyl- $\beta$-alanine; DGTS, diacylglyceroltrimethyhomoserine; DHA docosahexaenoic acid; ; GL, glycolipids DPA - docosapentaenoic acid; EPA - eicosapentaenoic acid; LCPUFA - long chain polyunsaturated fatty acid; MGDG - monogalactosyldiacylglycerol; NL, non-polar or neutral lipids; PA, phosphatidic acid; PC, phosphatidylcholine; PDAT, phospholipid: diacylglycerol acyltransferase; PE, phosphatidyethanolamine; PG, phosphatidylglycerol; PI, phosphatidylinositol; PL, phospholipids; PS, phosphatidylserine; SQDG, sulfoquinovosyldiacylglycerol; TAG, triacylglycerol.

\section{Declarations}

\section{Ethics approval and consent to participate}

Not applicable 
Not applicable

\section{Availability of data and materials}

All data supporting the conclusions of this article are included within the article and its additional files.

\section{Competing interests}

The authors declare that they have no competing interests.

\section{Funding}

O.S., J.A.N. and R.P.H receive grand-aided support from the Biotechnology and Biological Sciences Research Council (BBSRC, UK), in the form of Institute Strategic Programme Grants BBS/E/C/000I0420 and BBS/E/C/00005207. M.L.H, J.A.N. and O.S were funded by a BBSRC Longer and Larger Grant (BB/L002957/1). C.E., R.S. and O.S received funding from BBSRC UK Research and Innovation grant $\mathrm{BB} / \mathrm{N010388/1.}$

\section{Authors' contribution}

OS, RPH and MLH designed the research experiments and wrote the paper; $\mathrm{MH}, \mathrm{RPH}, \mathrm{CE}$ and RS performed the experiments and analysed the data; OS, RPH and JAN supervised the study, coordinated the writing and edited the drafts of the article. All authors read and approved the final manuscript. R.P.H. and M.L.H. contributed equally to this work.

\section{Acknowledgements}

We thank Kirsty Halsey of the Rothamsted Bioimaging group for the confocal images.

\section{References}

1. Liu Q, Siloto RMP, Lehner R, Stone SJ, Weselake RJ. Acyl-CoA:diacylglycerol acyltransferase: Molecular biology, biochemistry and biotechnology. Prog Lipid Res. 2012;51(4):350-377.

2. Zulu NN, Zienkiewicz K, Vollheyde K, Feussner I. Current trends to comprehend lipid metabolism in diatoms. Prog Lipid Res. 2018;70:1-16.

3. Li-Beisson Y, Thelen JJ, Fedosejevs E, Harwood JL. The lipid biochemistry of eukaryotic algae. Prog Lipid Res. 2019;74:31-68.

4. Hu Q, Sommerfeld M, Jarvis E, Ghirardi M, Posewitz M, Seibert M, Darzins A. Microalgal triacylglycerols as feedstocks for biofuel production: perspectives and advances. Plant J. 2008;54(4):621-639.

5. Cui Y, Thomas-Hall SR, Schenk PM. Phaeodactylum tricornutum microalgae as a rich source of omega-3 oil: Progress in lipid induction techniques towards industry adoption. Food Chem. 2019;297 
6. Zienkiewicz K, Du Z-Y, Ma W, Vollheyde K, Benning C. Stress-induced neutral lipid biosynthesis in microalgae - Molecular, cellular and physiological insights. Biochim Biophys Acta (BBA) - Mol and Cell Biol Lipids. 2016;1861(9, Part B):1269-1281 .

7. Ohlrogge JB, Jaworski JG. Regulation of fatty acid synthesis. Annu Rev Plant Physiol Plant Mol Biol. 1997;48:109-136.

8. Saha S, Enugutti B, Rajakumari S, Rajasekharan R. Cytosolic triacylglycerol biosynthetic pathway in oilseeds. Molecular cloning and expression of peanut cytosolic diacylglycerol acyltransferase. Plant Physiol. 2006;141(4):1533-1543.

9. Hernandez ML, Whitehead L, He ZS, Gazda V, Gilday A, Kozhevnikova E, Vaistij FE, Larson TR, Graham IA. A cytosolic acyltransferase contributes to triacylglycerol synthesis in sucrose-rescued Arabidopsis seed oil catabolism mutants. Plant Physiol. 2012;160(1):215-225.

10. Ahmad I, Sharma AK, Daniell H, Kumar S. Altered lipid composition and enhanced lipid production in green microalga by introduction of brassica diacylglycerol acyltransferase 2. Plant Biotechnol J. 2015,13(4):540-550.

11. Sanjaya, Miller R, Durrett TP, Kosma DK, Lydic TA, Muthan B, Koo AJK, Bukhman YV, Reid GE, Howe GA et al. Altered Lipid Composition and Enhanced Nutritional Value of Arabidopsis Leaves following Introduction of an Algal Diacylglycerol Acyltransferase 2. Plant Cell. 2013;25(2):677-693.

12. Chen $Y-C$. The biomass and total lipid content and composition of twelve species of marine diatoms cultured under various environments. Food Chem. 2012;131(1):211-9.

13. Vieler A, Wu GX, Tsai CH, Bullard B, Cornish AJ, Harvey C, et al. Genome, Functional Gene Annotation, and Nuclear Transformation of the Heterokont Oleaginous Alga Nannochloropsis oceanica CCMP1779. Plos Genetics. 2012;8(11).

14. Guiheneuf F, Leu S, Zarka A, Khozin-Goldberg I, Khalilov I, Boussiba S. Cloning and molecular characterization of a novel acyl-CoA:diacylglycerol acyltransferase 1-like gene (PtDGAT1) from the diatom Phaeodactylum tricornutum. FEBS J. 2011;278(19):3651-3666.

15. Zienkiewicz K, Zienkiewicz A, Poliner E, Du ZY, Vollheyde K, Herrfurth C, Marmon S, Farre EM, Feussner I, Benning C. Nannochloropsis, a rich source of diacylglycerol acyltransferases for engineering of triacylglycerol content in different hosts. Biotechnol Biofuels. 2017;10(1):8.

16. Hung $\mathrm{CH}$, Ho MY, Kanehara K, Nakamura Y. Functional study of diacylglycerol acyltransferase type 2 family in Chlamydomonas reinhardtii. FEBS Lett. 2013;587(15):2364-2370.

17. Liu J, Han DX, Yoon K, Hu Q, Li YT. Characterization of type 2 diacylglycerol acyltransferases in Chlamydomonas reinhardtii reveals their distinct substrate specificities and functions in triacylglycerol biosynthesis. Plant J. 2016;86(1):3-19.

18. Wagner M, Hoppe K, Czabany T, Heilmann M, Daum G, Feussner I, Fulda M. Identification and characterization of an acyl-CoA:diacylglycerol acyltransferase 2 (DGAT2) gene from the microalga $O$. tauri. Plant Physiol Bioch. 2010;48(6):407-416.

19. Gong Y, Guo X, Wan X, Liang Z, Jiang M. Triacylglycerol accumulation and change in fatty acid content of four marine oleaginous microalgae under nutrient limitation and at different culture ages. 
J Basic Microb. 2013;53(1):29-36.

20. Cui YL, Zheng GT, Li XQ, Lin HZ, Jiang P, Qin S. Cloning and characterization of a novel diacylglycerol acyltransferase from the diatom Phaeodactylum tricornutum. J Applied Phycol. 2013;25(5):15091512.

21. Dinamarca J, Levitan O, Kumaraswamy GK, Lun DS, Falkowski PG. Overexpression of a diacylglycerol acyltransferase gene in Phaeodactylum tricornutum directs carbon towards lipid biosynthesis. J Phycol. 2017;53(2):405-414.

22. Manandhar-Shrestha K, Hildebrand M. Characterization and manipulation of a DGAT2 from the diatom Thalassiosira pseudonana: Improved TAG accumulation without detriment to growth, and implications for chloroplast TAG accumulation. Algal Res. 2015;12:239-48.

23. Hamilton ML, Haslam RP, Napier JA, Sayanova O. Metabolic engineering of Phaeodactylum tricornutum for the enhanced accumulation of omega-3 long chain polyunsaturated fatty acids. Metab Eng. 2014;22:3-9.

24. Hamilton M, Haslam RP, Sayanova O, Napier JA. Metabolic engineering of diatoms for the enhanced production of high value lipids. Eur J Phycol. 2015;50:38-38.

25. Hamilton ML, Powers S, Napier JA, Sayanova O. Heterotrophic production of omega-3 Long-Chain Polyunsaturated Fatty Acids by trophically converted marine diatom Phaeodactylum tricornutum. Mar Drugs. 2016;14(3).

26. Pudney A, Gandini C, Economou CK, Smith R, Goddard P, Napier JA, Spicer A, Sayanova O. Multifunctionalizing the marine diatom Phaeodactylum tricornutum for sustainable co-production of omega-3 long chain polyunsaturated fatty acids and recombinant phytase. Sci Rep-UK. 2019;9:1-10.

27. Gong Y, Zhang J, Guo X, Wan X, Liang Z, Hu CJ, Jiang M. Identification and characterization of PtDGAT2B, an acyltransferase of the DGAT2 acyl-Coenzyme A: Diacylglycerol acyltransferase family in the diatom Phaeodactylum tricornutum. FEBS Lett. 2013;587(5):481-487.

28. Abida H, Dolch L-J, Mei C, Villanova V, Conte M, Block MA, Finazzi G, Bastien O, Tirichine L, Bowler C et al. Membrane glycerolipid remodeling triggered by nitrogen and phosphorus starvation in Phaeodactylum tricornutum. Plant Physiol. 2015;167(1):118-136.

29. Apt KE, KrothPancic PG, Grossman AR. Stable nuclear transformation of the diatom Phaeodactylum tricornutum. Mol Gen Genet. 1996;252(5):572-579.

30. Seo S, Jeon H, Hwang S, Jin E, Chang KS. Development of a new constitutive expression system for the transformation of the diatom Phaeodactylum tricornutum. Algal Res. 2015;11:50-54.

31. Popko J, Herrfurth C, Feussner K, Ischebeck T, Iven T, Haslam R, Hamilton M, Sayanova O, Napier J, Khozin-Goldberg I et al. Metabolome analysis reveals betaine lipids as major source for triglyceride formation, and the accumulation of sedoheptulose during nitrogen-starvation of phaeodactylum tricornutum. Plos One 2016;11(10):e0164673

32. Breuer G, Lamers PP, Martens DE, Draaisma RB, Wijffels RH. The impact of nitrogen starvation on the dynamics of triacylglycerol accumulation in nine microalgae strains. Bioresource Technol. 2012;124:217-226. 
33. Dolch L-J, Marechal E. Inventory of fatty acid desaturases in the pennate diatom Phaeodacty/um tricornutum. Mar Drugs. 2015;13(3):1317-1339.

34. Yoon K, Han DX, Li YT, Sommerfeld M, Hu Q. Phospholipid:diacylglycerol acyltransferase is a multifunctional enzyme involved in membrane lipid turnover and degradation while synthesizing triacylglycerol in the unicellular green microalga Chlamydomonas reinhardtii. Plant Cell. 2012; 24(9):3708-3724.

35. Guillard RR, Ryther JH. Studies of marine planktonic diatoms. I. Cyclotella nana Hustedt, and Detonula confervacea (Cleve) Gran. Can J Microbiol. 1962;8:229-239.

36. Kroth PG. Genetic transformation: a tool for study protein targeting in diatoms. Methods Mol. Biol. (Clifton, NJ). 2007;390:257.

37. Cooksey KE, Guckert JB, Williams SA, Callis PR. Fluorometric-determination of the neutral lipidcontent of microalgal cells using Nile Red. J Microbiol Methods. 1987;6(6):333-345.

38. Siaut M, Heijde M, Mangogna M, Montsant A, Coesel S, Allen A, Manfredonia A, Falciatore A, Bowler C. Molecular toolbox for studying diatom biology in Phaeodactylum tricornutum. Gene. 2007;406(12):23-35.

39. Govender T, Ramanna L, Rawat I, Bux F. BODIPY staining, an alternative to the Nile Red fluorescence method for the evaluation of intracellular lipids in microalgae. Bioresource Technol. 2012;114:507511.

40. Ruiz-Lopez N, Haslam RP, Napier JA, Sayanova O. Successful high-level accumulation of fish oil omega-3 long-chain polyunsaturated fatty acids in a transgenic oilseed crop. Plant $\mathrm{J}$. 2014;77(2):198-208.

\section{Figures}


a

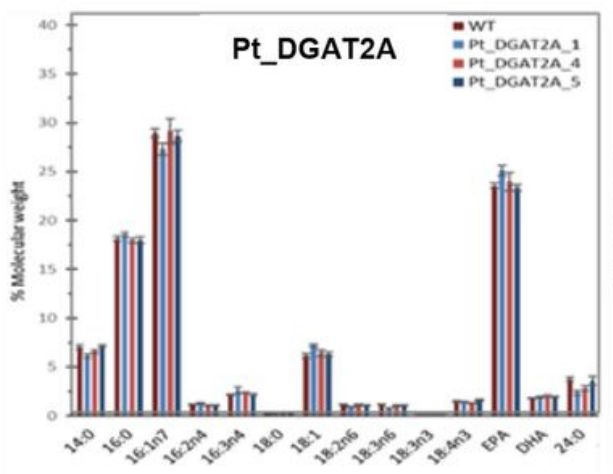

b
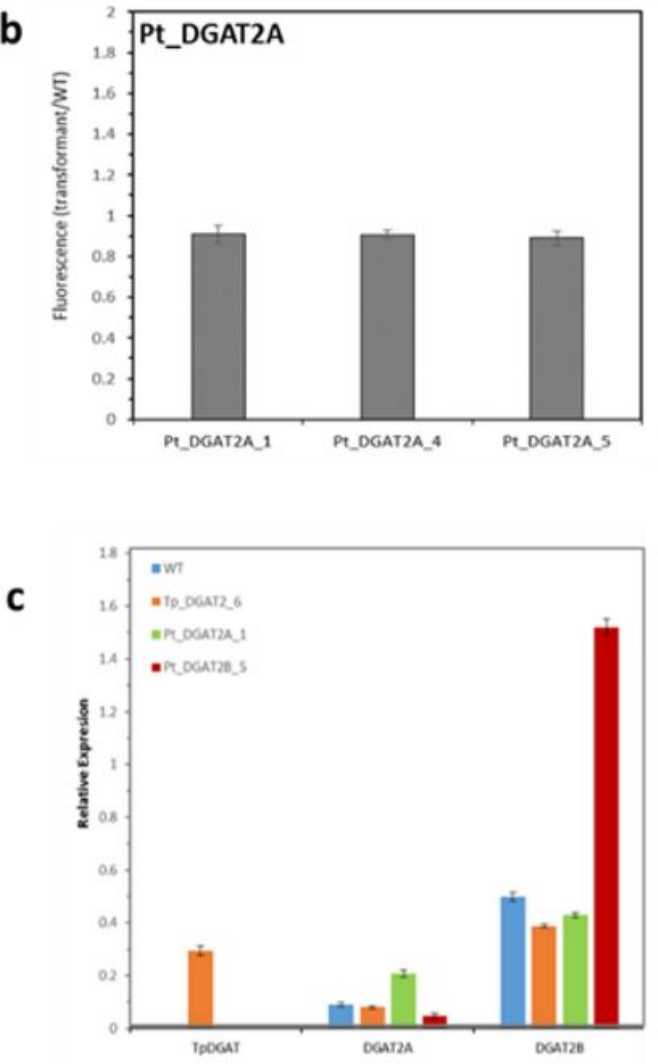
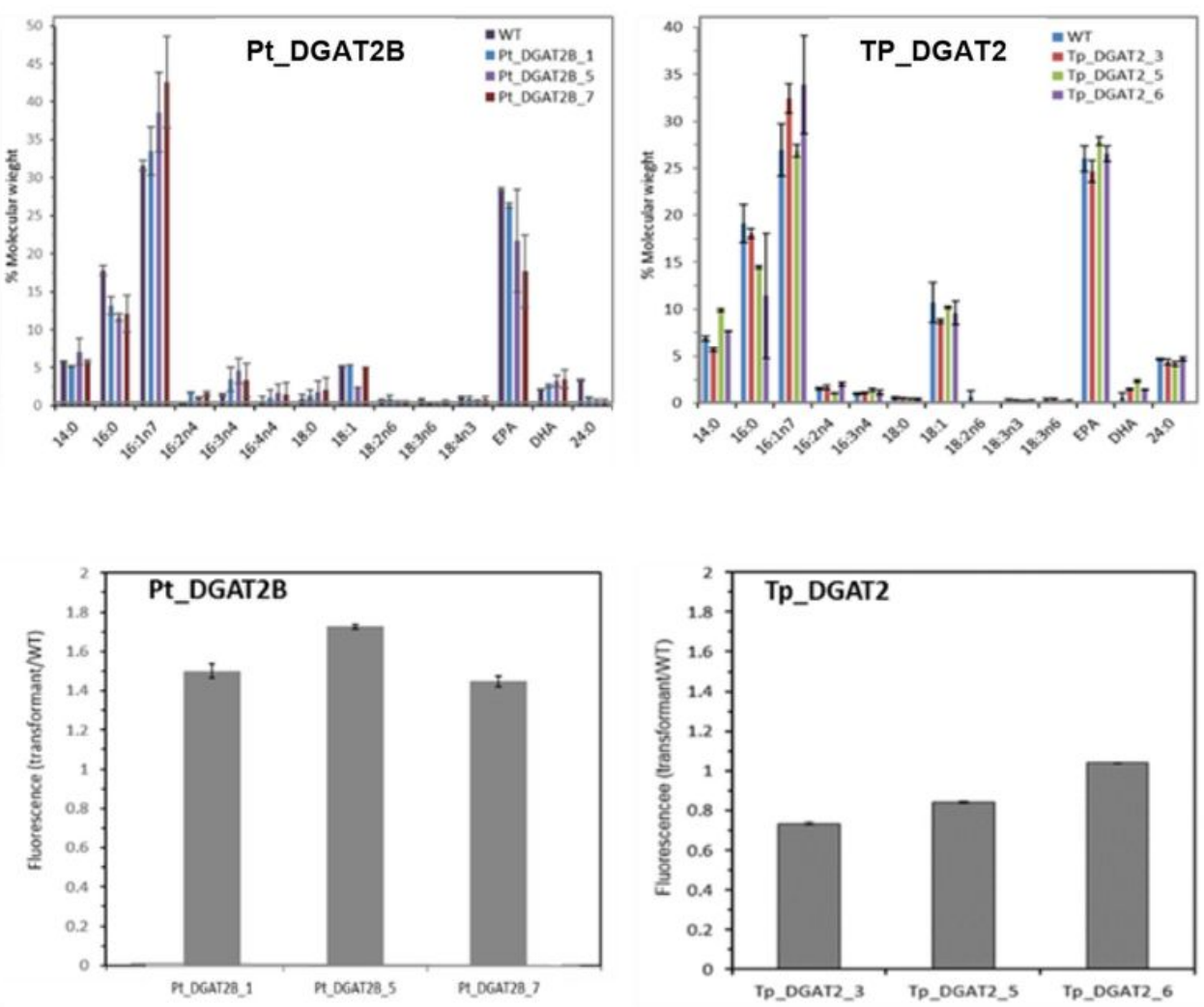

\section{Figure 1}

Overexpression of DGAT2 isoforms in P. tricornutum. a Fatty acid composition (Mol \%) of independent transgenic lines overexpressing DGAT2 genes during $S$ phase. Each measurement is the average of three technical replicas ( \pm standard error). b Neutral lipid content of transgenic cells overexpressing different DGAT2 isoforms assessed by Nile Red fluorescence. Each data point represents an average of three biological replicas. c Quantitative RT-PCR analysis of the transcript levels of DGAT2 isoforms. Transgenic algal cultures overexpressing TpDGAT2, PtDGAT2A and PtDGAT2B were grown under continuous light for three days in standard F/2 medium. Relative expression levels were normalized to that of the housekeeping gene RPS. 

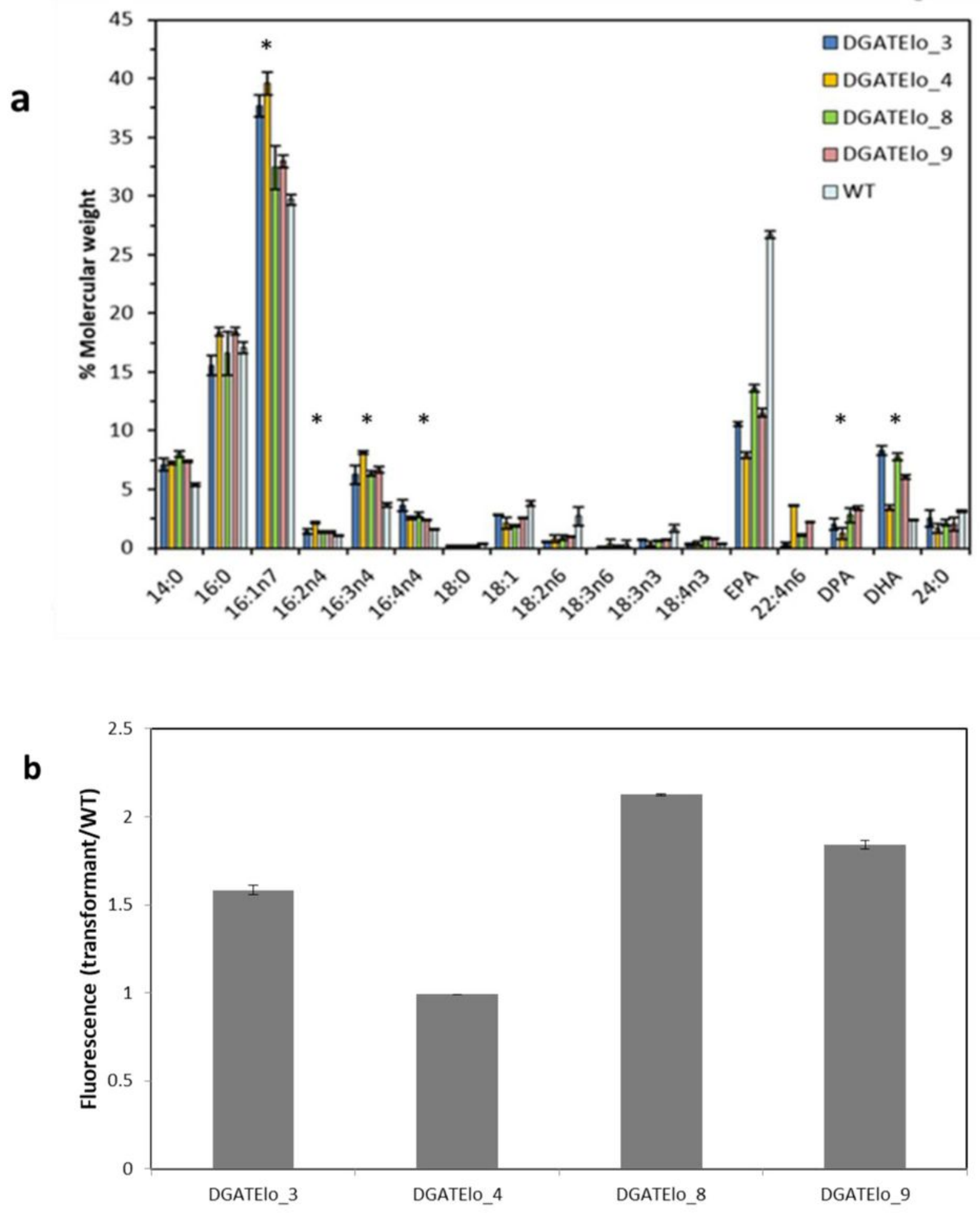

Figure 2

Functional characterization of transgenic P. tricornutum lines coexpressing DGAT2B and OtElo5. a Fatty acid composition of WT and DGATElo5 cells in the $S$ phase of growth in $F / 2$ media. Each measurement is the average of three biological replicas ( \pm standard error). b Neutral lipid content of DGATElo transgenic clones assessed by Nile Red fluorescence. Relative level of fluorescence in the transformants compared 
to WT cells. Values are the average of three replicated experiments. Error bars indicate standard error. Fatty acids which are more abundant in transgenic cells compared to WT are indicated by an asterisk $\left(^{\star}\right)$.
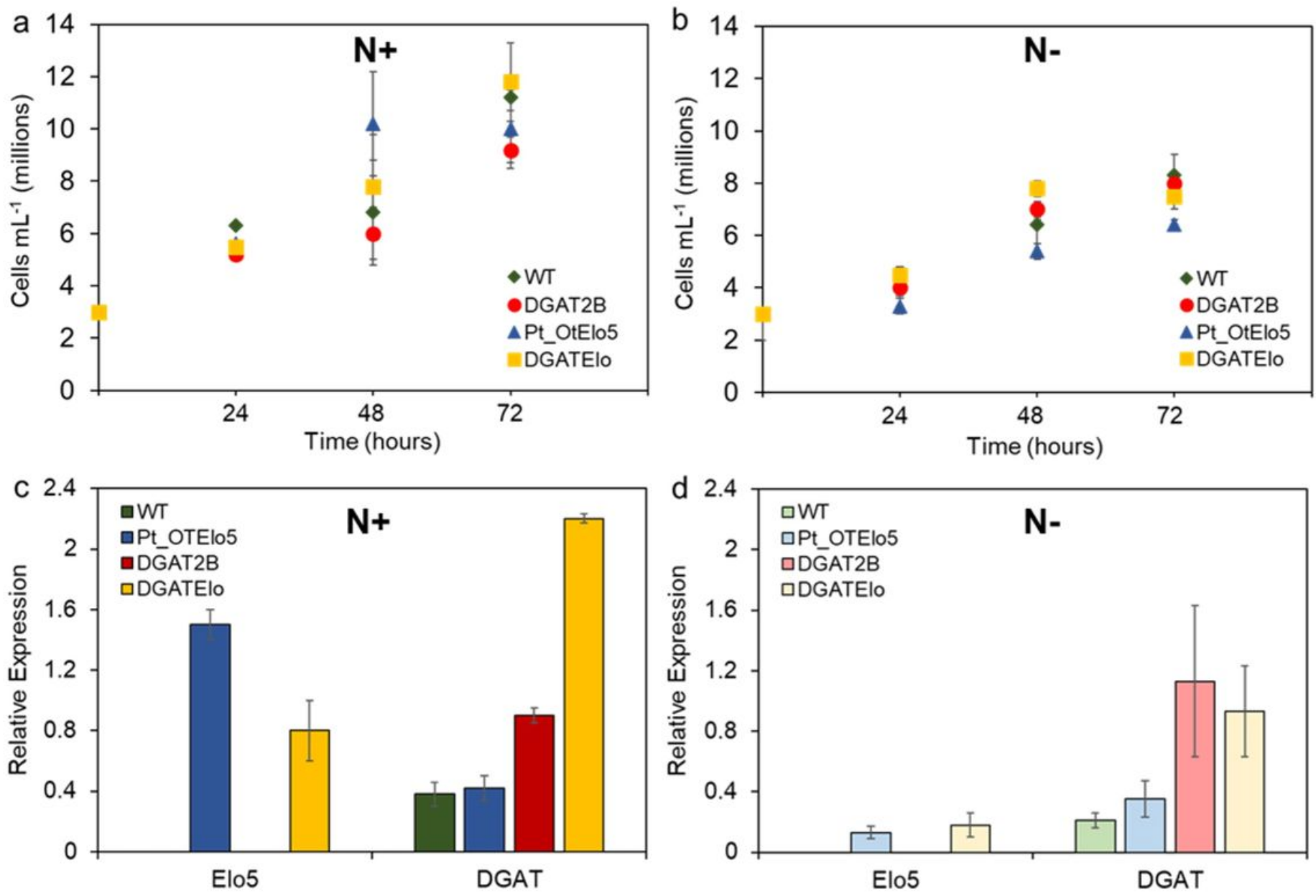

\section{Figure 3}

Cell growth and relative expression of transgenes in WT and transgenic P. tricornutum strains. Panels a and $\mathrm{b}$ cellular growth of WT and transgenic Pt_OtElo5, DGAT2B and DGATElo strains. Cells grown under $\mathrm{N}+$ and $\mathrm{N}$-conditions were harvested for lipid analysis where indicated. Values are the average of four experiments ( \pm standard error). Panels $\mathrm{c} \& d$ the relative expression of OtElo5 and DGAT2B transcript levels analysed by qRT-PCR in transformants growing under $\mathrm{N}+$ and $\mathrm{N}$ - conditions. Algal cultures were grown under continuous light for 72 hours in standard $\mathrm{F} / 2$ medium containing nitrogen $(\mathrm{N}+)$ or in $\mathrm{N}$ deprived ( $\mathrm{N}$-) conditions. Relative transcript abundance was normalized to that of housekeeping gene RPS and calculated using the 2_ $\Delta \Delta C T$ method. Values are the average of three replicated experiments. Error bars indicate standard error. 

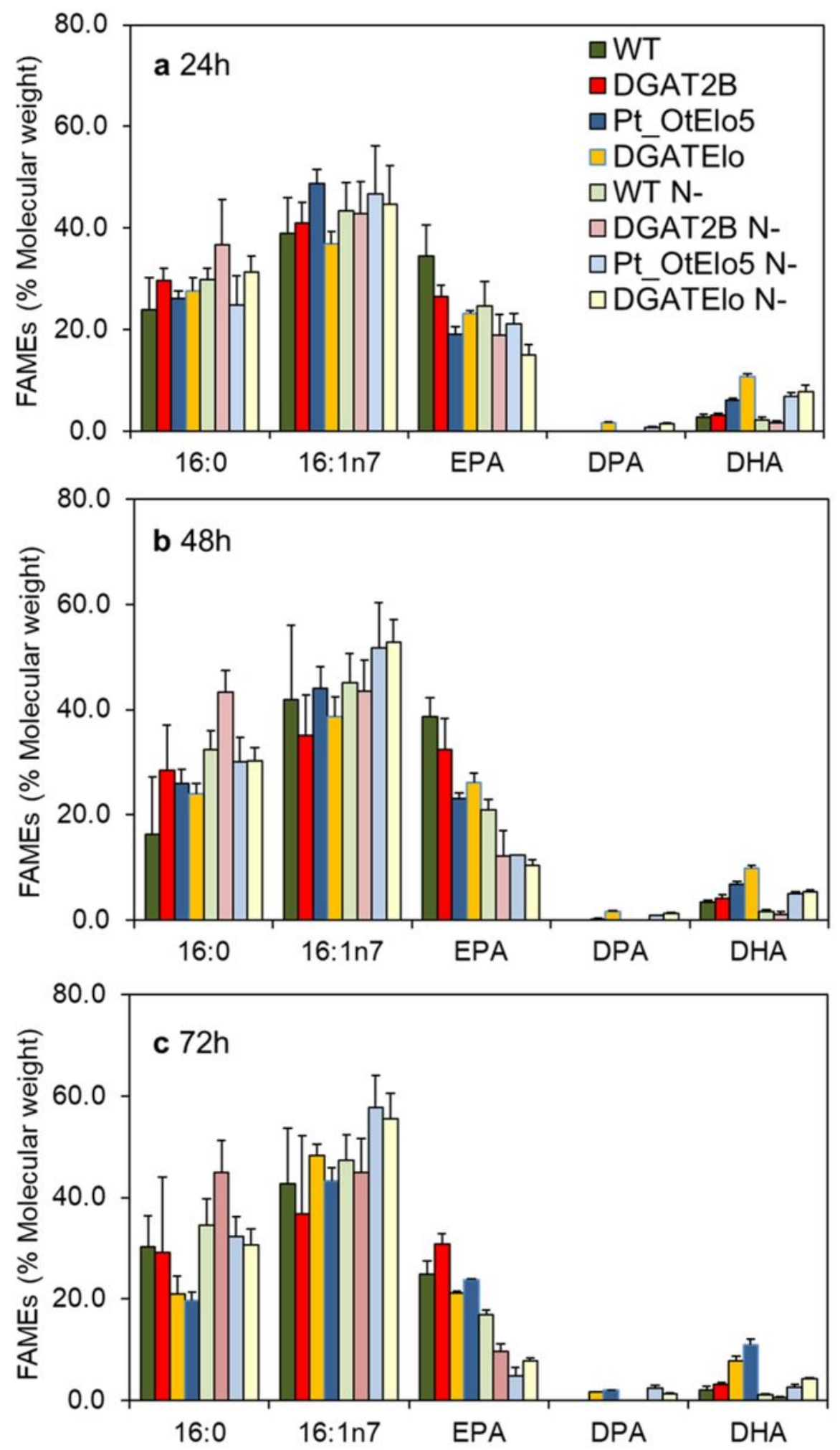

Figure 4

Fatty acid composition (Mol \%) of WT and transgenic (Pt_OtElo5, DGAT2B \& DGATElo) P. tricornutum. Cells cultuvated in $\mathrm{N}$-replete $(\mathrm{N}+$, dark bars) and $\mathrm{N}$-deplete $(\mathrm{N}$-, pale bars) medium at 24 (a), 48 (b) and 72 (c) hours. Each measurement is the average of minimum four technical replicates. Error bars indicate standard error. 

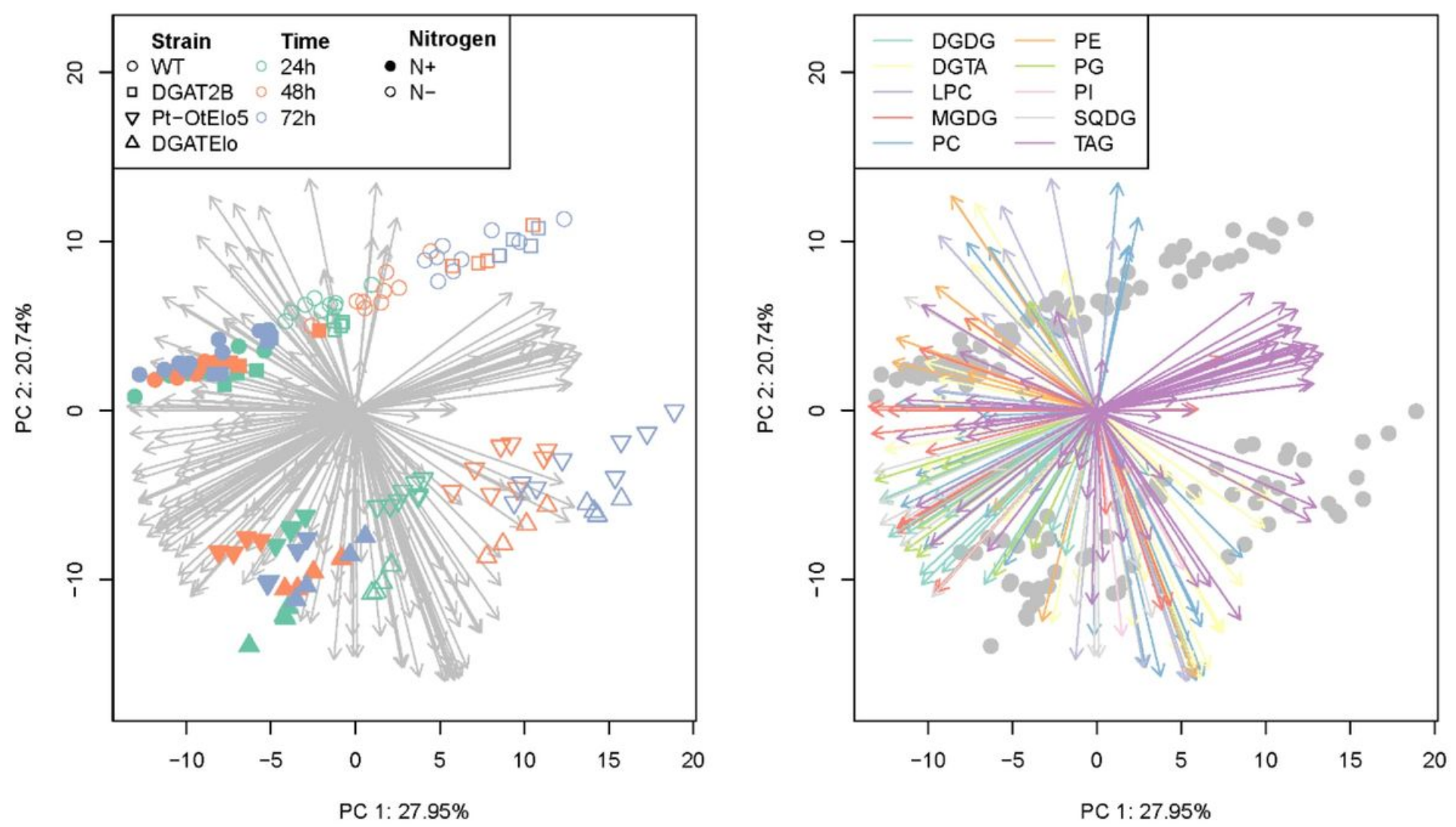

Figure 5

PCA plots of targetted lipidomic analysis (ESI-MS/MS) of P. tricornutum strains. Variate biplots of the first two principal axes derived from a principal component analysis characterising the response of cellualr lipids over time of P. tricornutum (WT, Pt_OtElo5, DGAT2B \& DGATElo) lines grown in N-replete and Ndeplete conditions of the log transformed data are shown. In both panels, points represent the treatment variates and are coloured in either by (a) treatment combinations (strain, time in hours and nitrogen treatment) or (b) lipid group (DGDG, MGDG, SQDG, PC, DGTA, LPC, PE, PI, PG, and TAG). The arrows represent the loadings associated with each principal vector. 


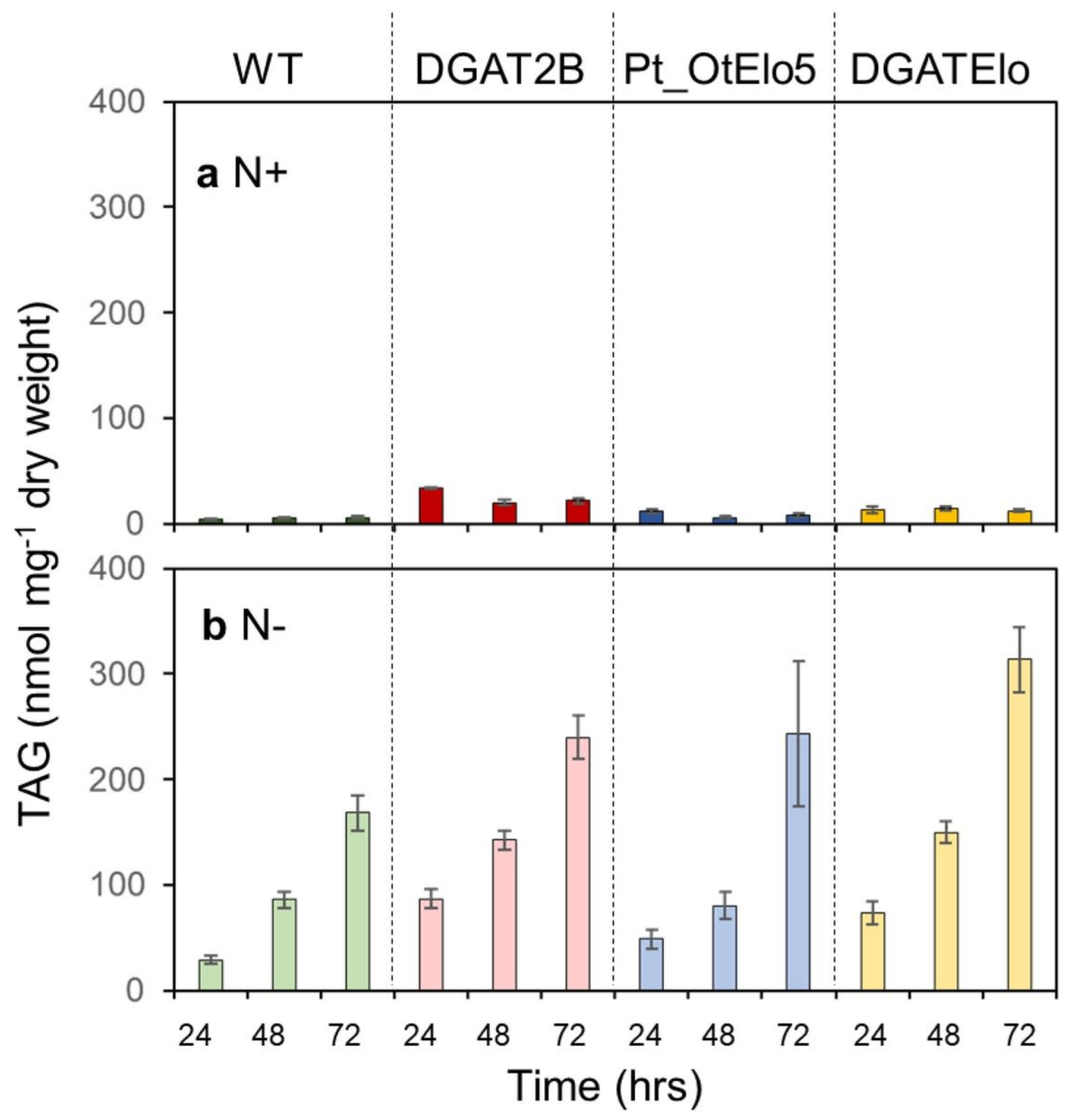

Figure 6

Quantitative analysis of TAG accumulation in P. tricornutum cells over a 72 hour time course. WT and transgenic (Pt_OtElo5, DGAT2B \& DGATElo) P. tricornutum lines were grown in (a) N-replete (N+) or (b) Ndeplete $(\mathrm{N}-)$ conditions. Total TAG content was determined by ESI-MS/MS analysis. Each measurement is the average of minimum four technical replicates. Error bars indicate standard error. 

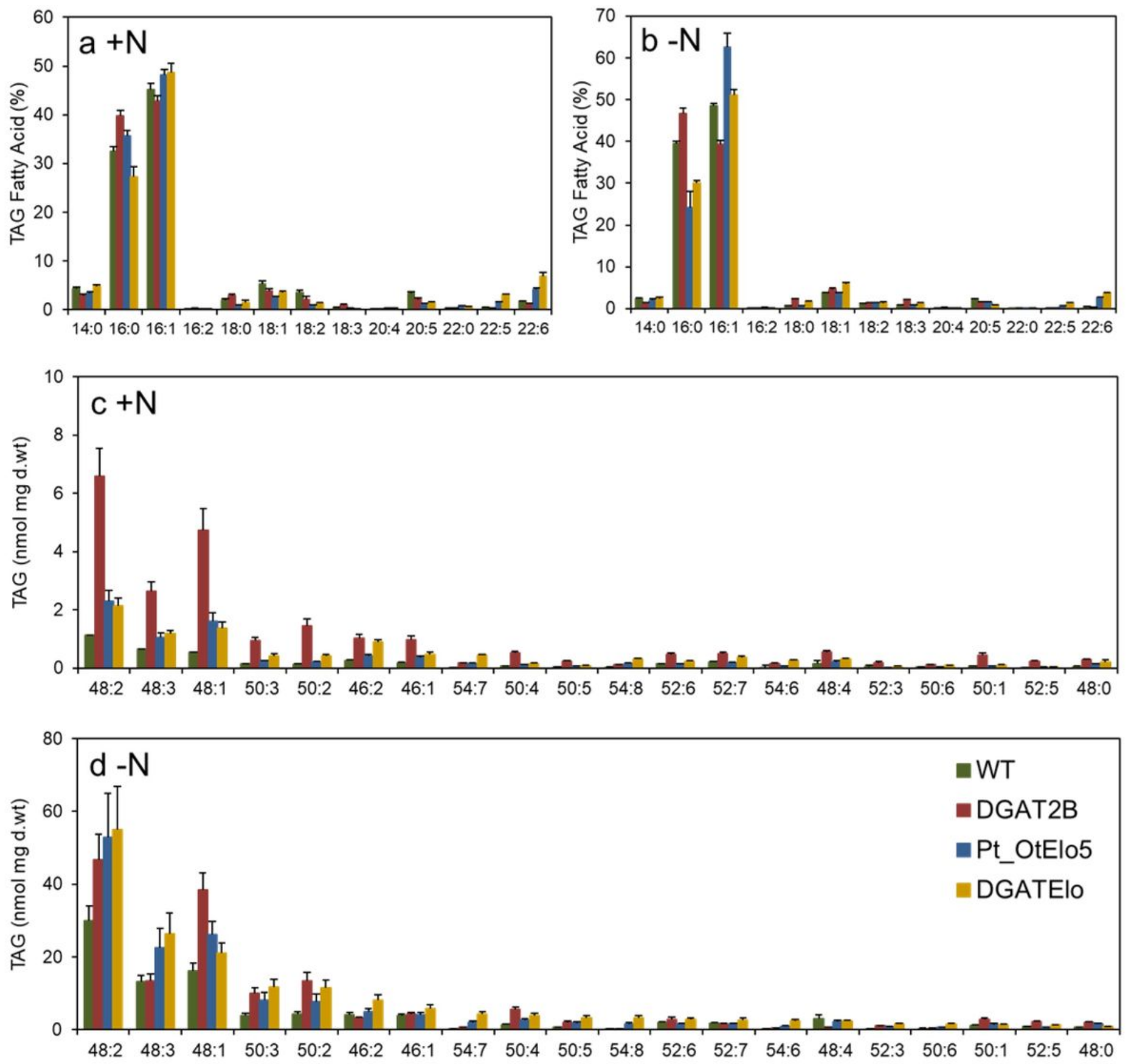

\section{Figure 7}

Compositional analysis of fatty acids and molecular species in TAG for P. tricornutum cells. Lipids were analysed after 72 hours of cultivation in N-replete $(+\mathrm{N})$ and $\mathrm{N}$-deplete $(-\mathrm{N})$ medium. Panels $(\mathrm{a} \& \mathrm{~b})$ TAG fatty acid composition; Panels (c \& d) TAG molecular species composition. Each measurement represents the average of at least four technical replicates. Error bars indicate standard error. Asterisks indicate dominant TAG species, which are significantly different between strains and those which are significantly altered by $\mathrm{N}$ stress. Black asterisks indicate TAG species containing 16:0 and 16:1 fatty acids; green 
asterisks indicate TAG species containing C16-C18 fatty acids; and blue asterisks indicate TAG species containing 20:5, 22:5 and 22:6.
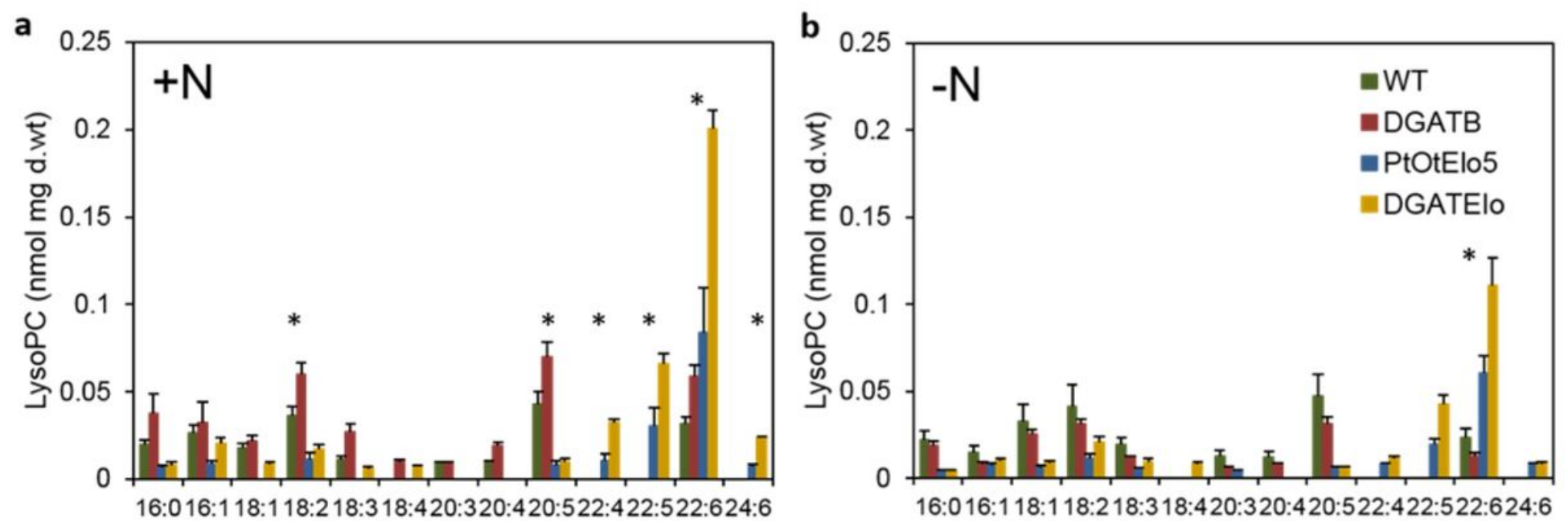

\section{Figure 8}

Targeted ESI-MS/MS analysis of Lyso-PC in WT and transgenic P. tricornutum. Lipids were analysed in WT, Pt_OtElo5, DGAT2B and DGATElo cells grown in N-replete $(+\mathrm{N})$ and $\mathrm{N}$-deplete $(-\mathrm{N})$ medium at $72 \mathrm{~h}$. Each measurement represents the average of at least four technical replicates. Error bars indicate standard error. FA species that are significantly different to WT, new species or increased under N stress are denoted by an asterisk $\left(^{\star}\right)$.

\section{Supplementary Files}

This is a list of supplementary files associated with this preprint. Click to download.

- Haslamadditionalfiles.pdf 